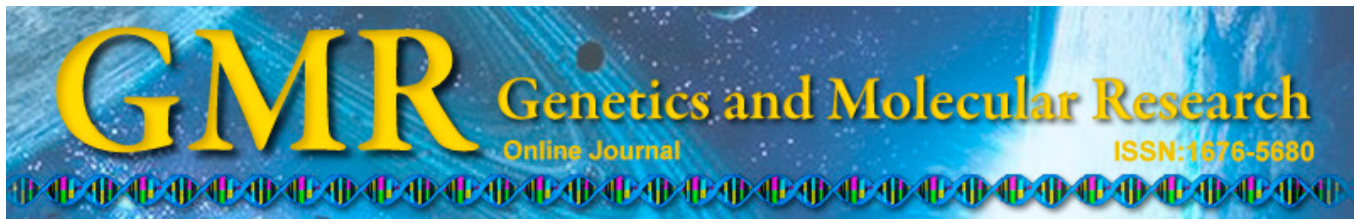

\title{
Genetic diversity of cultivated and wild tomatoes revealed by morphological traits and SSR markers
}

\section{R. Zhou, Z. Wu, X. Cao and F.L. Jiang}

Key Laboratory of Horticultural Plant Biology and Germplasm Innovation in East China, Ministry of Agriculture, College of Horticulture, Nanjing Agricultural University, Nanjing, China

Corresponding author: $\mathrm{Z}$. Wu

E-mail:wzh@njau.edu.cn

Genet. Mol. Res. 14 (4): 13868-13879 (2015)

Received January 6, 2015

Accepted June 23, 2015

Published October 29, 2015

DOI http://dx.doi.org/10.4238/2015.October.29.7

\begin{abstract}
In the current study, morphological traits and molecular markers were used to assess the genetic diversity of 29 cultivated tomatoes, 14 wild tomatoes and seven introgression lines. The three components of the principal component analysis (PCA) explained $78.54 \%$ of the total morphological variation in the 50 tomato genotypes assessed. Based on these morphological traits, a three-dimensional PCA plot separated the 50 genotypes into distinct groups, and a dendrogram divided them into six clusters. Fifteen polymorphic genomic simplesequence repeat (genomic-SSR) and 13 polymorphic expressed sequence tag-derived SSR (EST-SSR) markers amplified 1115 and 780 clear fragments, respectively. Genomic-SSRs detected a total of 64 alleles, with a mean of 4 alleles per primer, while EST-SSRs detected 52 alleles, with a mean of 4 alleles per primer. The polymorphism information content was slightly higher in genomic-SSRs (0.49) than in EST-SSRs (0.45). The mean similarity coefficient among the wild tomatoes was lower than the mean similarity coefficient among the cultivated tomatoes. The dendrogram based on genetic distance
\end{abstract}


divided the 50 tomato genotypes into eight clusters. The Mantel test between genomic-SSR and EST-SSR matrices revealed a good correlation, whereas the morphological matrices and the molecular matrices were weakly correlated. We confirm the applicability of EST-SSRs in analyzing genetic diversity among cultivated and wild tomatoes. High variability of the 50 tomato genotypes was observed at the morphological and molecular level, indicating valuable tomato germplasm, especially in the wild tomatoes, which could be used for further genetic studies.

Key words: Genetic diversity analysis; Morphological traits; Molecular marker; Wild tomato

\section{INTRODUCTION}

Tomato (Solanum lycopersicum L., formerly Lycopersicon esculentum Mill.) is one of the most economically important vegetables widely grown for its fruit, and consumed either fresh or processed. The annual global production of tomato exceeds 161 million tons (FAO, 2012).

Cultivated tomatoes typically have low genetic diversity due to population bottlenecks (Rick, 1976), and intensive selection of a few desired traits during domestication has led to further loss of genetic diversity among the commercial tomato varieties (Williams and Clair, 1993). The genetic base of tomato varieties is narrow, which makes it difficult to identify different varieties using molecular markers. Fortunately, the model tomato vegetable from the family Solanceae has abundant genomic information, including a complete reference genome sequence and a rapidly developing comparative genomic database (http://solgenomics.net/). Therefore, many molecular markers generated from the sequence database have been successfully applied for identification and genetic diversity analysis of tomato cultivars (Park et al., 2004; García-Martínez et al., 2006; Benor et al., 2008; Korir et al., 2014).

There are 16 wild species of tomato, including S. habrochaites, S. pennellii, S. pimpinellifolium, S. cheesmaniae, S. galapagense, S. peruvianum, S. corneliomulleri, S. chilense, $S$. chmielewskii, S. arcanum, S. neorickii, S. huaylasense, S. lycopersicoides, S. ochranthum, S. jugandifolium, and S. sitiens (Knapp et al., 2009; Bedinger et al., 2011). These species in the tomato clade are all diploid with the same chromosome number $(2 \mathrm{n}=24)$ and are considered to have evolved primarily by genic changes rather than large-scale chromosomal rearrangements (Anderson et al., 2010). The wild species of tomato harbor many valuable genes, which may have been lost among cultivated tomatoes. As wild species can enlarge the gene pool of cultivated species, wild species are very useful in breeding programs as sources of genetic variability (Hanson et al., 2007). It is of great importance to have a clear understanding of the genetic diversity and relationship between cultivated tomatoes and their wild species for effective conservation, classification, and further utilization of tomato germplasm resources.

Morphological description and classification is a traditional approach used to quantify genetic differences, and is often used for genetic diversity analysis (Khadivi-Khub et al., 2008; Terzopoulos and Bebeli, 2008; Nikoumanesh et al., 2011). However, morphological traits are easily altered by environmental conditions; thus, quantification of genotypic variation is not always possible (Cooke et al., 2003). By comparison, molecular markers are generally recognized as a reliable method for the identification of genotypes, such as amplified 
fragment length polymorphisms (AFLPs) (Park et al., 2004), randomly amplified polymorphic DNA (RAPD) (Nikoumanesh et al., 2011; Cao et al., 2015), and simple-sequence repeats or microsatellites (SSRs) (Benor et al., 2008; Wohrmann et al., 2011). Among these molecular markers, SSR markers are very popular due to their characteristics of high reproducibility, co-dominance, and polymorphism (Powell et al., 1996). EST-SSRs, the SSRs derived from expressed sequence tags (ESTs), are rapidly developing markers and have been used for genetic diversity analysis of a wide range of plant species (Jia et al., 2007; Caruso et al., 2008; Huang et al., 2010; Korir et al., 2014). Moreover, using a combination of morphological and molecular markers to identify plant genetic diversity has become more common (Khadivi-Khub et al., 2008; Terzopoulos and Bebeli, 2008; Mazzucato et al., 2010; Nikoumanesh et al., 2011).

However, evaluation of the genetic relationship of cultivated and wild tomatoes using genomic-SSR and EST-SSR has not been undertaken to date. Taking the phenotypic diversity among the cultivated and wild tomatoes into account, a dual strategy was employed in this study to investigate the genetic diversity of the tomato germplasm based on both morphological and genetic markers (genomic-SSRs and EST-SSRs). The aim was to test the applicability of EST-SSRs in tomato genetic diversity assessments and clarify the variability of cultivated and wild tomatoes at the morphological and molecular level.

\section{MATERIAL AND METHODS}

\section{Plant material}

A total of 29 cultivated tomatoes (S. lycopersicum), 14 wild tomatoes, and seven introgression lines (ILs) developed from a cross between S. pennellii and S. lycopersicum were donated by the Tomato Genetics Resource Center (TGRC, Davis, CA, USA). The details of the tomato material are shown in Table 1.

\section{Morphological data}

Details of the nine morphological traits recorded for the tomatoes are shown in Table S1. Briefly, the traits recorded were growth habit, plant height $(\mathrm{cm})$, stem diameter $(\mathrm{mm})$, hypocotyl color, leaf shape, leaf area $\left(\mathrm{cm}^{2}\right)$, leaf length $(\mathrm{cm})$, leaf width $(\mathrm{cm})$, and pubescence of stem and leaf. These descriptors mainly conform to the guidelines of the International Plant Genetic Resources Institute (Mazzucato et al., 2010). The flower and fruit traits were also investigated, but these data were not used for further analysis as some wild tomatoes in our collection were self-incompatible.

\section{Genotypic data}

Total genomic DNA was isolated from young leaves of 50-day-old seedlings using a DNA extraction kit (Takara, Shanghai, China) following the manufacturer protocol, and the quality and quantity of DNA was assessed against known concentrations of unrestricted lambda DNA on a $1 \%$ agarose gel.

Genomic-SSR and EST-SSR markers were commercially provided by the Biotech Company (Invitrogen, Shanghai, China). Details of the 15 genomic-SSR and 13 EST-SSR primers used in the study were shown in Table S2. Amplification reactions were performed 
in a total volume of $20 \mu \mathrm{L}$, containing $11.6 \mu \mathrm{L} \mathrm{ddH_{2 }} \mathrm{O}, 1 \mu \mathrm{L}$ gDNA (20 ng), $1 \mu \mathrm{L}$ forward and reverse primers $(10 \mu \mathrm{M}), 2 \mu \mathrm{L} \mathrm{10X}$ buffer, $1.2 \mu \mathrm{L} \mathrm{MgCl}_{2}(25 \mathrm{mM}), 2 \mu \mathrm{L}$ dNTPs $(2.5 \mathrm{mM})$, and $0.2 \mu \mathrm{L}$ Taq DNA polymerase $(5 \mathrm{U} / \mu \mathrm{L})$ (Takara). The reactions were carried out on 96-well plates in an Authorized Thermal Cycler (Eppendorf, Hamburg, Germany) with the following program: $94^{\circ} \mathrm{C}$ for $3 \mathrm{~min}$, then 35 cycles of $94^{\circ} \mathrm{C}$ for $45 \mathrm{~s}, 54^{\circ} \mathrm{C}$ for $45 \mathrm{~s}$, and $72^{\circ} \mathrm{C}$ for $1 \mathrm{~min}$, with a final step at $72^{\circ} \mathrm{C}$ for $5 \mathrm{~min}$. Annealing temperature was changed based on the primers used (Table S2). The PCR products were verified by $8 \%$ polyacrylamide gel electrophoresis and visualized by silver staining. The DL 500-bp DNA marker (Takara) was included on each gel to estimate band size. Electrophoresis was run at $100 \mathrm{~V}$ and its expendable time depended on the size of each marker. Amplification and verification was repeated twice for every polymorphic marker to ensure reliability of the data.

\begin{tabular}{|c|c|c|c|c|c|c|c|}
\hline Number & Code & Taxon & Type & Number & Code & Taxon & Type \\
\hline 1 & LA4090 & Solanum lycopersicum & Cultivated & 26 & LA3668 & S. lycopersicum & $\overline{\text { Cultivated }}$ \\
\hline 2 & LA1019 & S. lycopersicum & Cultivated & 27 & LA3847 & S. lycopersicum & Cultivated \\
\hline 3 & LA 1023 & S. lycopersicum & Cultivated & 28 & LA4355 & S. lycopersicum & Cultivated \\
\hline 4 & LA 1222 & S. lycopersicum & Cultivated & 29 & LA4440 & S. lycopersicum & Cultivated \\
\hline 5 & LA1500 & S. lycopersicum & Cultivated & 30 & LA1223 & S. habrochaites & Wild \\
\hline 6 & LA1563 & S. lycopersicum & Cultivated & 31 & LA1777 & S. habrochaites & Wild \\
\hline 7 & LA 1698 & S. lycopersicum & Cultivated & 32 & LA3915 & Introgression line & - \\
\hline 8 & LA1994 & S. lycopersicum & Cultivated & 33 & LA3916 & Introgression line & - \\
\hline 9 & LA1996 & S. lycopersicum & Cultivated & 34 & LA3917 & Introgression line & - \\
\hline 10 & LA2006 & S. lycopersicum & Cultivated & 35 & LA3918 & Introgression line & - \\
\hline 11 & LA2013 & S. lycopersicum & Cultivated & 36 & LA3919 & Introgression line & - \\
\hline 12 & LA2019 & S. lycopersicum & Cultivated & 37 & LA3920 & Introgression line & - \\
\hline 13 & LA2413 & S. lycopersicum & Cultivated & 38 & LA3932 & Introgression line & - \\
\hline 14 & LA2661 & S. lycopersicum & Cultivated & 39 & LA0716 & S. pennellii & Wild \\
\hline 15 & LA2662 & S. lycopersicum & Cultivated & 40 & LA1926 & S. pennellii & Wild \\
\hline 16 & LA2683 & S. lycopersicum & Cultivated & 41 & LA1598 & S. pimpinellifolium & Wild \\
\hline 17 & LA2706 & S. lycopersicum & Cultivated & 42 & LA2093 & S. pimpinellifolium & Wild \\
\hline 18 & LA2838 & S. lycopersicum & Cultivated & 43 & LA0923 & S. cheesmaniae & Wild \\
\hline 19 & LA3006 & S. lycopersicum & Cultivated & 44 & LA0483 & S. galapagense & Wild \\
\hline 20 & LA3120 & S. lycopersicum & Cultivated & 45 & LA1627 & S. galapagense & Wild \\
\hline 21 & LA3183 & S. lycopersicum & Cultivated & 46 & LA 0454 & S. peruvianum & Wild \\
\hline 22 & LA3320 & S. lycopersicum & Cultivated & 47 & LA 1274 & S. corneliomulleri & Wild \\
\hline 23 & LA3473 & S. lycopersicum & Cultivated & 48 & LA 1969 & S. chilense & Wild \\
\hline 24 & LA3475 & S. lycopersicum & Cultivated & 49 & LA 2748 & S. chilense & Wild \\
\hline 25 & LA3538 & S. lycopersicum & Cultivated & 50 & LA 2408 & S. lycopersicoides & Wild \\
\hline
\end{tabular}

\section{Data analysis}

The morphological data were used in principal component analysis (PCA) and cluster analysis in SPSS version 16.0 (SPSS Inc., Chicago, IL, USA). For genotypic data, the presence or absence of the band was scored as 1 or 0, respectively. Based on analysis of bands, the polymorphism information content (PIC) for each SSR was calculated with the PIC-CALC software, according to the formula:

$$
\mathrm{PIC}=1-\sum \mathrm{p}_{\mathrm{ij}}^{2}
$$

where, $p_{i j}$ is the frequency of the $\mathrm{i}^{\text {th }}$ allele of the $j$ marker (Weir, 1990). Pairwise genetic simi- 
larities $\left(S_{i j}\right)$ among accessions $i$ and $j$ were estimated using the similarity coefficient described by Nei and $\mathrm{Li}(1979)$ as follows:

$$
\mathrm{S}_{\mathrm{ij}}=2 \mathrm{~N}_{\mathrm{ij}} /\left(\mathrm{N}_{\mathrm{i}}+\mathrm{N}_{\mathrm{j}}\right)
$$

where, $N_{i j}$ is the number of bands present in the $i$ and $j$ cultivars, with $N_{i}$ and $N_{j}$ representing the number of bands present in cultivar $i$ and $j$, respectively. A dendrogram was generated using the unweighted pair group mean analysis method based on genetic distance with the NTSYS software. In addition, dissimilarity matrices were calculated by Mantel tests using the NTSYS software.

\section{RESULTS}

\section{Morphological characterization and clustering}

PCA of the morphological traits showed that the three components represented $78.54 \%$ of the total phenotypic variation (Table 2). The first component accounted for $47.46 \%$ of the phenotypic variation where the width, length, and area of leaf had the highest loadings. Leaf traits were predominant in the first component and contributed to most of the phenotypic variation. The second component, which featured plant height as the principal trait, explained an additional $20.78 \%$ of the phenotypic variation. Finally, the third component explained a further $10.30 \%$ of the variation in which hypocotyl color was predominant. The three-dimensional PCA plot shows the distribution of tomato genotypes based on the morphological traits (Figure 1). Cultivated tomatoes mainly clustered into two groups (S1, S2), along with the ILs. The wild tomatoes, except number 31 (S. habrochaites), clustered separately from the cultivated tomatoes.

Morphological cluster analysis showed six distinct clusters at a Euclidean distance of five (Figure 2). Cluster I included 14 cultivated tomatoes and four ILs; cluster II comprised ten wild tomatoes and three cultivated tomatoes; cluster III was composed of three wild tomatoes; cluster IV was composed of two cultivated tomatoes; cluster V comprised ten cultivars and three ILs; and cluster VI consisted of a single wild tomato (number 48).

\begin{tabular}{|c|c|c|c|}
\hline Morphological traits & $\mathrm{PC} 1$ & $\mathrm{PC} 2$ & $\mathrm{PC} 3$ \\
\hline Growth habit & 0.327 & -0.911 & -0.026 \\
\hline Plant height & -0.452 & 0.833 & -0.062 \\
\hline Stem diameter & 0.801 & 0.173 & 0.116 \\
\hline Hypocotyl color & 0.559 & -0.114 & 0.570 \\
\hline Pubescence of stem and leaf & 0.730 & -0.011 & 0.267 \\
\hline Leaf shape & 0.549 & 0.475 & 0.325 \\
\hline Leaf length & 0.885 & 0.187 & -0.224 \\
\hline Leaf width & 0.909 & 0.204 & -0.202 \\
\hline Leaf area & 0.750 & -0.007 & -0.563 \\
\hline Cumulative eigenvalues & 47.46 & 68.24 & 78.54 \\
\hline
\end{tabular}




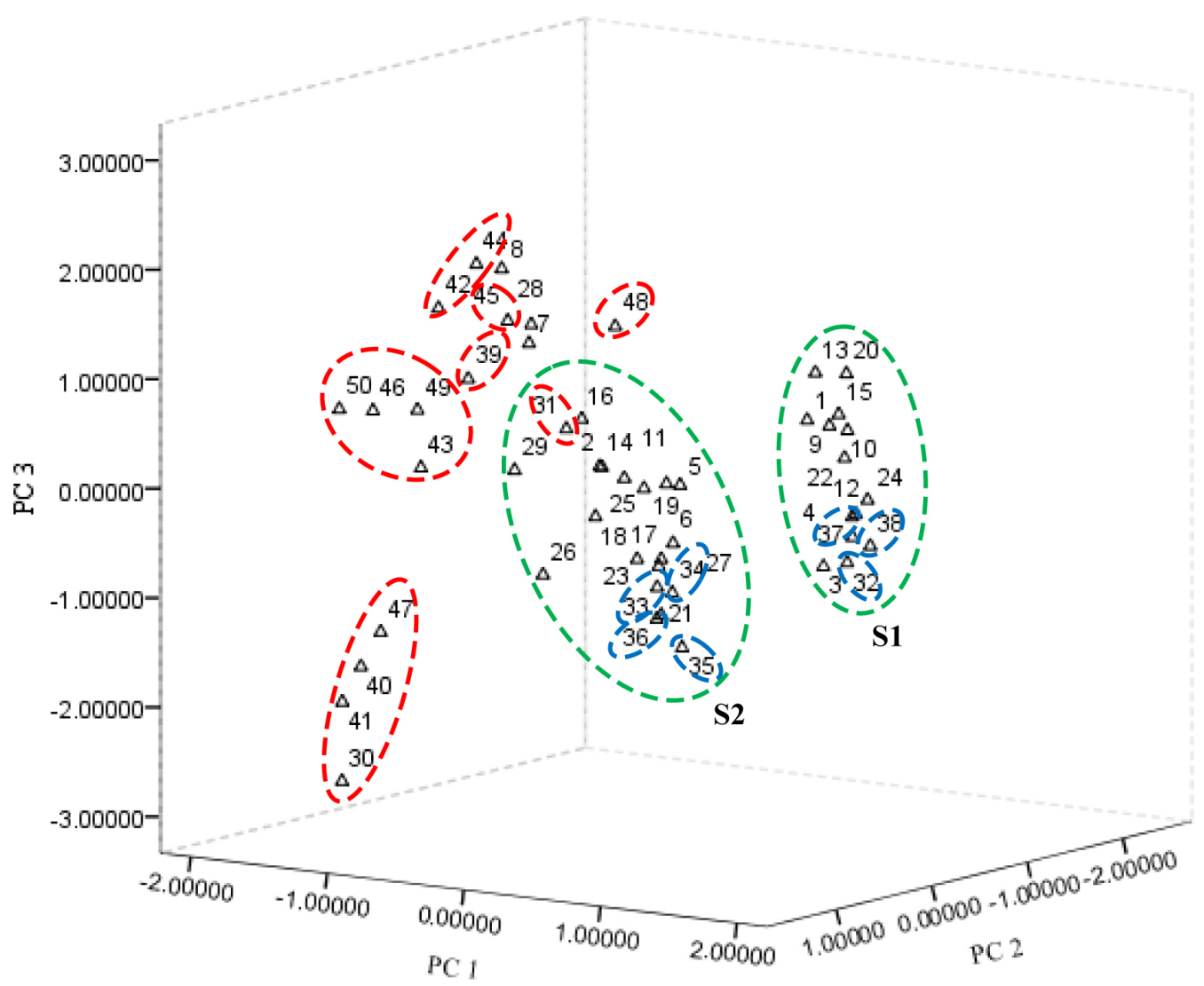

Figure 1. Three-dimensional principal component (PC) analysis plot of the 50 tomato genotypes based on morphological traits. Numbers correspond to the tomato genotypes in Table 1. The two sections (S1, S2) within the green dashed lines show the main distribution of the cultivated tomatoes in this study. Tomatoes within the blue dashed lines are introgression lines, while those within the red dashed lines are wild tomatoes.

\section{Allelic variation based on SSR markers}

The allelic variation of the genomic-SSR and EST-SSR markers is shown in Table 3. The number of clear fragments detected by 15 polymorphic genomic-SSRs in the 50 tomato genotypes was 1115, with a mean of 74 fragments per primer. Genomic-SSRs detected 64 alleles in total, with a mean of 4 alleles per primer and ranging from 2 to 8 . The 13 EST-SSR primers amplified clear polymorphic products in the 50 tomato genotypes, with 780 clear fragments and a mean of 60 fragments per primer. The number of alleles ranged from 2 to 7 , with a total of 52 alleles for all primers and a mean of 4 alleles per primer. DNA fingerprinting by the EST-SSR35 primer is shown in Figure S1. The mean PIC for the genomic-SSR loci was 0.49 , ranging from 0.08 to 0.80 , and the mean PIC for EST-SSR loci was 0.45 , ranging from 0.14 to 0.71 . Genomic-SSR and EST-SSR markers jointly amplified 1895 clear fragments with a mean of 68 fragments per primer. There were a total of 116 alleles, with a mean of 4 alleles per primer for the SSR markers. 


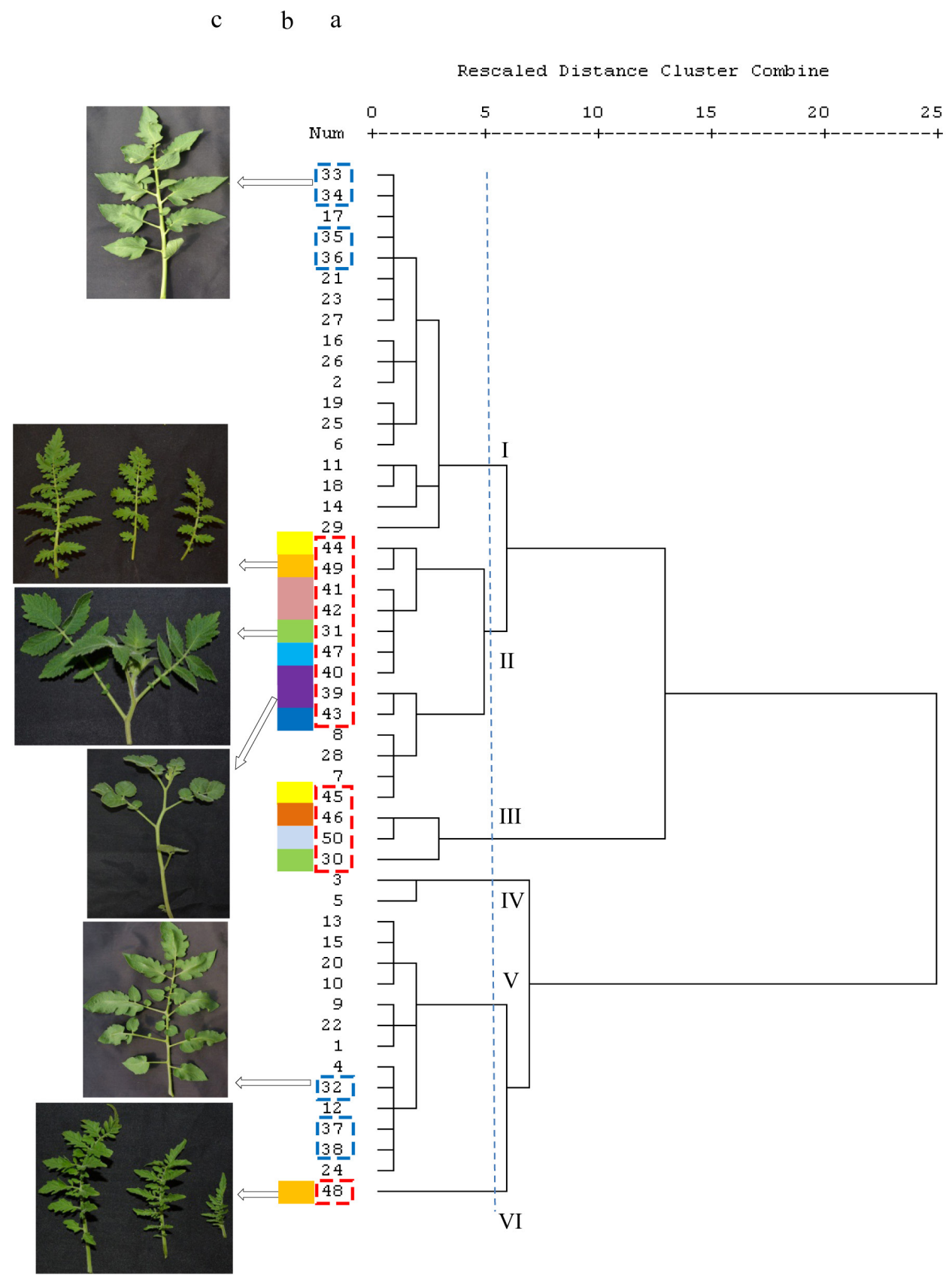

Figure 2. Cluster analysis of the 50 tomato genotypes based on morphological traits. The Roman numerals denote the six clusters. (a) Numbers correspond to the tomato genotypes in Table 1. Tomatoes within the blue dashed lines are introgression lines, while those within the red dashed lines are the wild tomatoes. (b) Different colored boxes to the left of the numbers represent different wild tomatoes. (c) Images of leaves from some tomato genotypes used in this study to show the leaf type variations. 
Table 3. Allelic variation revealed by the polymorphic genomic simple sequence repeat (genomic-SSR) and expressed sequence tag-derived SSR (EST-SSR) among the 50 tomato genotypes.

\begin{tabular}{lcrr}
\hline Item & Genomic-SSR & EST-SSR & In total \\
\hline Number of polymorphic primers & 15 & 13 & 28 \\
Total number of fragments & 1115 & 780 & 1895 \\
Mean number of fragments per primer pair & 74 & 60 & 68 \\
Total number of alleles & 64 & 52 & 4 \\
Mean number of alleles per primer pair & 4 & 416 & 4 \\
Polymorphism information content (range/mean) & $0.08-0.80 / 0.49$ & $0.14-0.71 / 0.45$ & $0.08-0.80 / 0.47$ \\
\hline
\end{tabular}

\section{Similarity coefficient and clustering of tomato genotypes based on SSR markers}

The similarity coefficients of the 50 tomato genotypes based on SSR markers are shown in Table S3 . These values ranged from 0.422 between $S$. lycopersicum and $S$. pennellii (numbers 5 and 40) to 0.994 between ILs (numbers 33 and 34), with a mean of 0.737 (Table S3). For the cultivated tomatoes, the minimum similarity coefficient was 0.724 (between numbers 6 and 16), with a mean of 0.845 (Table 4). For the ILs, the minimum and maximum similarity coefficients were 0.845 (between numbers 35 and 38) and 0.994 (between numbers 33 and 34), respectively (Table 4). The similarity coefficients of the wild tomatoes ranged from 0.457 (between numbers 40 and 41) to 0.836 (between numbers 43 and 44) (Table 4).

Genetic cluster analysis based on the genetic distance matrix is shown in Figure 3. There were eight clusters among all tomato genotypes at a distance coefficient of 0.66 . Cluster I comprised 36 genotypes, including the 29 cultivated tomatoes and seven ILs. The wild tomatoes clustered together in the lower section of the dendrogram in clusters II to VI. There was only the one wild tomato within cluster VIII.

Table 4. Minimum (min), maximum ( $\max$ ), and mean values of the similarity coefficient among all tomato genotypes, cultivated tomatoes, introgression lines, and wild tomatoes.

\begin{tabular}{lcccc}
\hline Similarity coefficient & Sample No. & Min & Max & Mean \\
\hline All tomato genotypes & 50 & 0.422 & 0.994 & 0.737 \\
Cultivated tomatoes & 29 & 0.724 & 0.994 & 0.845 \\
Introgression lines & 7 & 0.845 & 0.994 & 0.932 \\
Wild tomatoes & 14 & 0.457 & 0.836 & 0.627 \\
\hline
\end{tabular}

\section{Correlation between morphological, genomic-SSR, and EST-SSR analysis}

The Mantel test correlation coefficient $(r)$ was 0.710 between genomic-SSR and ESTSSR matrices (Table 5). Combined data from both sets of molecular markers shows a closer relationship to the data from only the genomic-SSR markers $(r=0.952)$ than that observed from only the EST-SSR markers $(r=0.887$; Table 5). However, the correlation coefficients between the morphological and molecular data were less than 0.2 (Table 5). 


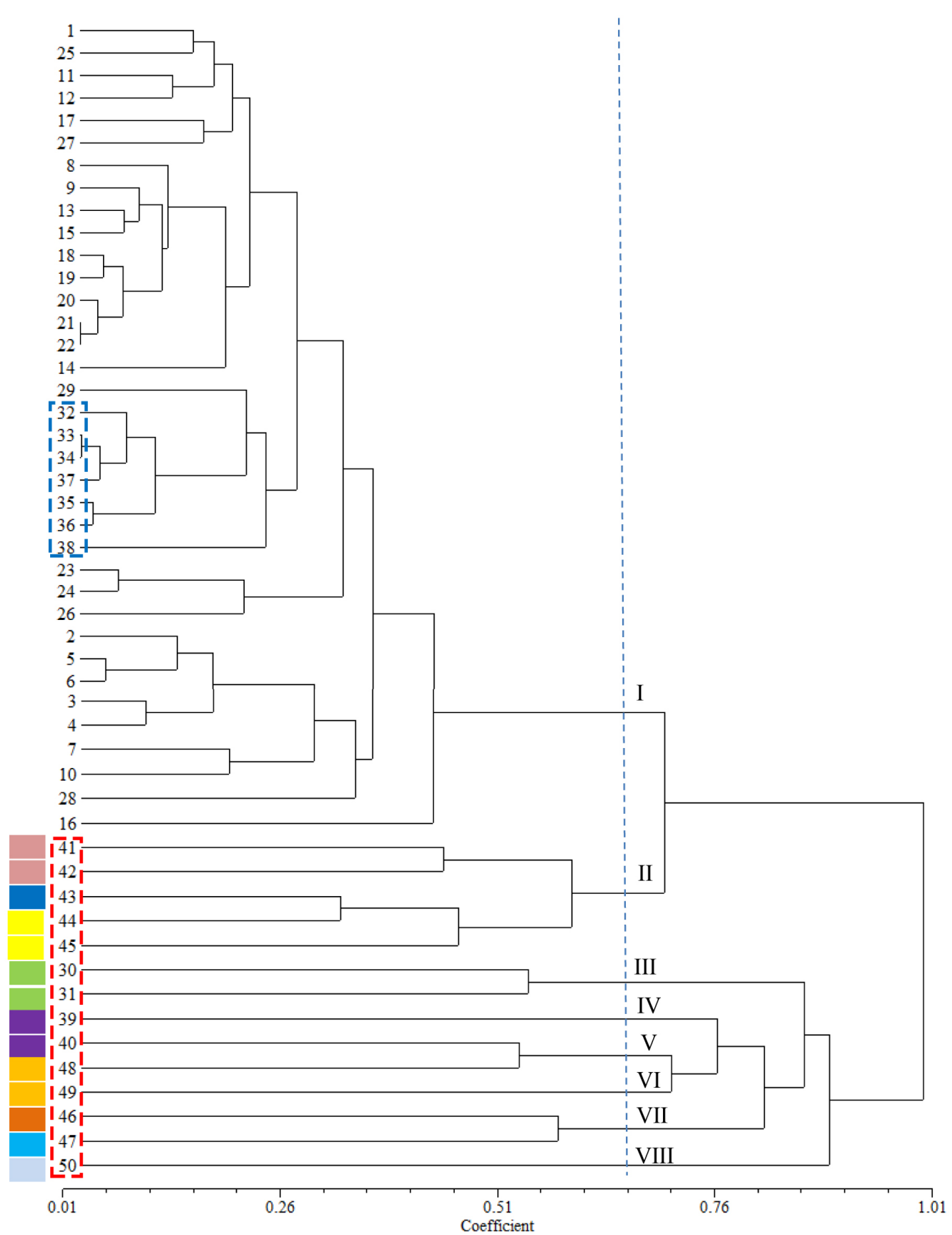

Figure 3. Dendrogram constructed using the unweighted pair group mean analysis method of the 50 tomato genotypes based on the genomic and expressed sequence tag simple-sequence repeat markers. The Roman numerals denote the eight clusters. Arabic numbers correspond to the tomato genotypes in Table 1. Tomatoes within the blue dashed lines are introgression lines, while those within the red dashed lines are the wild tomatoes. Different colored boxes to the left of the numbers represent different wild tomatoes. 
Table 5. Mantel test correlation coefficient between the morphological matrices and the molecular matrices of the 50 tomato genotypes.

\begin{tabular}{lcccc}
\hline Correlation coefficient & Morphology & Genomic-SSR & EST-SSR & Genomic-SSR and EST-SSR \\
\hline Morphology & 1.000 & - & - & - \\
Genomic-SSR & 0.157 & 1.000 & - & - \\
EST-SSR & 0.128 & 0.710 & 1.000 & - \\
Genomic-SSR and EST-SSR & 0.157 & 0.952 & 0.887 & 1.000
\end{tabular}

SSR, simple sequence repeat; EST, expressed sequence tag.

\section{DISCUSSION}

Using a combination of morphological traits and molecular markers has been shown to lead to more reliable conclusions in assessments of genetic diversity (Khadivi-Khub et al., 2008; Nikoumanesh et al., 2011; Acosta-Quezada et al., 2012). According to the morphological results presented in the current study, leaf variation is crucial for distinguishing wild tomatoes from cultivated tomatoes as a result of i) most of the wild tomatoes possess specific leaf characteristics; ii) the results of PCA showed leaf traits to be the major contributors in the first component. The morphological cluster analysis was effective for classifying the cultivated and wild tomatoes. Based on morphological traits, the 50 tomato genotypes were divided into six clusters with the wild tomatoes gathered in clusters II, III, and VI. The morphological cluster of some wild tomatoes was in good agreement with their botanical classification. For example, two tomato genotypes from S. pimpinellifolium (numbers 41 and 42) showed a close relationship in the morphological cluster. However, there was a large distance between the two tomato genotypes from S. chilense (numbers 48 and 49). This indicates that some wild tomatoes cannot be discriminated clearly based on morphological characteristics alone, as morphological characteristics can easily be affected by the environment.

The cluster analysis based on the genomic-SSR and EST-SSR markers was more effective in comparison to the morphological cluster analysis. The cultivated tomatoes and ILs comprised cluster I, while the wild tomatoes comprised the other seven clusters. The ILs were expected to cluster together with the cultivated tomatoes as the ILs have a genetic background of a S. lycopersicum chromosome with a single restriction fragment length polymorphism-defined S. pennelliichromosome segment. This also explains why the ILs showed a high similarity coefficient (0.932).

The EST-SSR markers were shown to be applicable for genetic diversity studies of tomato, which is in accordance with Muñoz-Falcón et al. (2011), Wohrmann et al. (2011), and Korir et al. (2014). In this study, the mean PIC of genomic-SSR markers was higher than the mean PIC for EST-SSR markers (0.49 and 0.45, respectively), which was also observed in eggplant (Muñoz-Falcón et al., 2011) and cucumber (Hu et al., 2011). High, medium, and low locus polymorphism is defined as PIC $>0.5,0.5>\mathrm{PIC}>0.25$, and $\mathrm{PIC}<0.25$, respectively (Xie et al., 2010). Therefore, in our case, genomic-SSR and EST-SSR markers detected medium locus polymorphism among the 50 tomato genotypes, indicating that both markers are of great utility for genetic diversity studies of tomatoes.

The Mantel test revealed a low correlation between morphological matrices and molecular matrices of the cultivated and wild tomatoes $(\mathrm{r}=0.157)$, which was also reported in Korean tomato varieties (Kwon et al., 2009), Greek tomato landraces (Terzopoulos and Bebeli, 2010), and almond genotypes and related Prunus species (Nikoumanesh et al., 2011). However, a good correlation between genomic-SSR and EST-SSR matrices $(r=0.710)$ was 
observed, which further confirms the applicability of EST-SSR markers for analyzing genetic diversity in tomatoes. Furthermore, morphological indictors provide fundamental phenotypic information of tomato plants that could be complementary to molecular markers.

Domestication of tomatoes with the aim of selecting a few desired traits has resulted in a narrow genetic base and low genetic diversity of cultivated tomatoes. This is indicated by the high similarity coefficient of the 29 cultivated tomatoes $(0.845)$ in this study. By contrast, wild tomatoes, with a lot of valuable genes, are important resources for improving the tolerance to both biotic and abiotic stress, such as S. habrochaites (Hanson et al., 2007; Sifres et al., 2011), which could expand the genetic base of cultivated tomatoes. The 14 wild tomatoes that were included in this study enriched the genetic diversity as indicated by i) their scattered distribution in the three-dimensional PCA plot (Figure 1); ii) the large genetic distance between them and the cultivated tomatoes (Figure 3); and iii) the lower similarity coefficient of the wild tomatoes $(0.627)$ as compared to the similarity coefficient of the cultivated tomatoes.

In the current study, we used morphological descriptors and molecular markers, including genomic-SSR and EST-SSR markers, to assess the diversity of cultivated and wild tomatoes. Our results indicate that EST-SSR markers are effective in identifying tomato genotypes and analyzing genetic diversity of cultivated and wild tomatoes. Considerable genetic diversity among the cultivated and wild tomatoes was observed at both the morphological and molecular levels, which is of importance for germplasm classification, management, and further utilization.

\section{Conflicts of interest}

The authors declare no conflict of interest.

\section{ACKNOWLEDGMENTS}

Research supported by grants from the Priority Academic Program Development of Jiangsu Higher Education Institutions (PAPD), the National Undergraduate Innovative Experimentation Program (\#NUIEP, 111030723), and the Research Innovation Program for College Graduates of Jiangsu Province (\#CXZZ12-0285).

\section{Supplementary material}

\section{REFERENCES}

Acosta-Quezada PG, Vilanova S, Martínez-Laborde JB and Prohens J (2012). Genetic diversity and relationships in accessions from different cultivar groups and origins in the tree tomato (Solanum betaceum Cav.). Euphytica 187: 87-97.

Anderson LK, Covey PA, Larsen LR, Bedinger P, et al. (2010). Structural differences in chromosomes distinguish species in the tomato clade. Cytogenet. Genome Res. 129: 24-34.

Bedinger PA, Chetelat RT, McClure B, Moyle LC, et al. (2011). Interspecific reproductive barriers in the tomato clade: opportunities to decipher mechanisms of reproductive isolation. Sex. Plant Reprod. 24: 171-187.

Benor S, Zhang M, Wang Z and Zhang H (2008). Assessment of genetic variation in tomato (Solanum lycopersicum L.) inbred lines using SSR molecular markers. J. Genet. Genomics 35: 373-379.

Cao X, Wu Z, Zhou R, Jiang FL, et al. (2015). A novel random amplified polymorphic DNA-based strategy for genetic diversity analysis and identification of tomatoes. Genet. Mol. Res. 14: 1650-1661.

Caruso M, Federici CT and Roose ML (2008). EST-SSR markers for asparagus genetic diversity evaluation and cultivar identification. Mol. Breed. 21: 195-204. 
Cooke RJ, Bredemeijer GMM, Ganal MW, Peeters R, et al. (2003). Assessment of the uniformity of wheat and tomato varieties at DNA microsatellite loci. Euphytica 132: 331-341.

FAO (2012). FAO Statistical Yearbook 2012. Food and Agriculture Organization (FAO) of the United Nations. http:// faostat.fao.org/site/339/default.aspx. Accessed August 2013.

García-Martínez S, Andreani L, García-Gusano M, Geuna F, et al. (2006). Evaluation of amplified fragment length polymorphism and simple sequence repeats for tomato germplasm fingerprinting: utility for grouping closely related traditional cultivars. Genome 49: 648-656.

Hanson PM, Sitathani K, Sadashiva AT, Yang RY, et al. (2007). Performance of Solanum habrochaites LA1777 introgression line hybrids for marketable tomato fruit yield in Asia. Euphytica 158: 167-178.

Hu J, Wang L and Li J (2011). Comparison of genomic SSR and EST-SSR markers for estimating genetic diversity in cucumber. Biol. Plantarum 55: 577-580.

Huang Y, Li F and Chen K (2010). Analysis of diversity and relationships among Chinese orchid cultivars using EST-SSR markers. Biochem. Syst. Ecol. 38: 93-102.

Jia XP, Shi YS, Song YC, Wang GY, et al. (2007). Development of EST-SSR in foxtail millet (Setaria italica). Genet. Resour. Crop Evol. 54: 233-236.

Khadivi-Khub A, Zamani Z and Bouzari N (2008). Evaluation of genetic diversity in some Iranian and foreign sweet cherry cultivars by using RAPD molecular markers and morphological traits. Hortic. Environ. Biotechnol. 49: 188-196.

Knapp S, Spooner DM and Peralta I (2009). Taxonomy of wild tomatoes and their relatives (Solanum sect. Lycopersicoides, sect. Juglandifolia, sect. Lycopersicon; Solanaceae). Syst. Bot. 84: 1-186.

Korir NK, Diao W, Tao R, Li X, et al. (2014). Genetic diversity and relationships among different tomato varieties revealed by EST-SSR markers. Genet. Mol. Res. 13: 43-53.

Kwon YS, Park SG and Yi SI (2009). Assessment of genetic variation among commercial tomato (Solanum lycopersicum L.) varieties using SSR markers and morphological characteristics. Genes Genom. 31: 1-10.

Mazzucato A, Ficcadenti N, Caioni M, Mosconi P, et al. (2010). Genetic diversity and distinctiveness in tomato (Solanum lycopersicum L.) landraces: the Italian case study of 'A pera Abruzzese'. Sci. Hortic. 125: 55-62.

Muñoz-Falcón JE, Vilanova S, Plazas M and Prohens J (2011). Diversity, relationships, and genetic fingerprinting of the Listada de Gandía eggplant landrace using genomic SSRs and EST-SSRs. Sci. Hortic. 129: 238-246.

Nei M and Li WH (1979). Mathematical model for studying genetic variation in terms of restriction endonucleases. Proc. Natl. Acad. Sci. U.S.A. 76: 5269-5273.

Nikoumanesh K, Ebadi A, Zeinalabedini M and Gogorcena Y (2011). Morphological and molecular variability in some Iranian almond genotypes and related Prunus species and their potentials for rootstock breeding. Sci. Hortic. 129: 108-118.

Park YH, West MA and St. Clair DA (2004). Evaluation of AFLPs for germplasm fingerprinting and assessment of genetic diversity in cultivars of tomato (Lycopersicon esculentum L.). Genome 47: 510-518.

Powell W, Morgante M, Andre C, Hanafey M, et al. (1996). The comparison of RFLP, RAPD, AFLP and SSR (microsatellite) markers for germplasm analysis. Mol. Breed. 2: 225-238.

Rick CM (1976). Tomato Lycopersicon esculentum (Solanaceae). In: Evolution of crop plants (Simmonds NW, ed.). Longman Group Ltd, London, 268-273.

Sifres A, Blanca J and Nuez F (2011). Pattern of genetic variability of Solanum habrochaites in its natural area of distribution. Genet. Resour. Crop Evol. 58: 347-360.

Terzopoulos PJ and Bebeli PJ (2008). DNA and morphological diversity of selected Greek tomato (Solanum lycopersicum L.) landraces. Sci. Hortic. 116: 354-361.

Terzopoulos PJ and Bebeli PJ (2010). Phenotypic diversity in Greek tomato (Solanum lycopersicum L.) landraces. Sci. Hortic. 126: 138-144.

Weir BS (1990). Genetic data analysis: methods for discrete population genetics data. Sinauer Associates, Inc., Sunderland.

Williams CE and Clair DA (1993). Phenetic relationships and levels of variability detected by restriction fragment length polymorphism and random amplified polymorphic DNA analysis of cultivated and wild accessions of Lycopersicon esculentum. Genome 36: 619-630.

Wohrmann T, Guicking D, Khoshbakht K and Weising K (2011). Genetic variability in wild populations of Prunus divaricata Ledeb. in northern Iran evaluated by EST-SSR and genomic SSR marker analysis. Genet. Resour. Crop Evol. 58: 1157-1167.

Xie WG, Zhang XQ, Cai HW, Liu W, et al. (2010). Genetic diversity analysis and transferability of cereal EST-SSR markers to orchard grass (Dactylis glomerata L). Biochem. Syst. Ecol. 38: 740-749. 